\title{
DESIGN AND DEVELOPMENT OF THE SNS RING VACUUM INSTRUMENTATION AND CONTROL SYSTEMS*
}

\author{
H.C. Hseuh, L.A. Smart and J.Y. Tang ${ }^{\dagger}$ \\ Collider-Accelerator Department, BNL, Upton, NY 11973, USA
}

\section{Abstract}

BNL is undertaking the design, construction and commissioning of the Spallation Neutron Source (SNS) accumulator ring and the beam transport lines [1]. Ultrahigh vacuum of $10^{-9}$ Torr is required in the accumulator ring to minimize beam-gas ionization, a contributing factor to the e-p instability observed in a few high-intensity proton storage rings. All vacuum instrumentation must be capable of local and remote operation to achieve a reliable vacuum system, especially in this extremely high intensity accelerator. The design and development of the SNS ring vacuum instrumentation and control through the Experimental Physics and Industrial Control System (EPICS) distributed real-time software tools are presented.

\section{INTRODUCTION}

The design of the instrumentation and control system for the SNS accumulator ring vacuum system was based on the architecture for the RHIC vacuum system [2, 3], a versatile system, operational for over five years, with a few device model replacements to comply with SNS standards. The control system software is currently being developed on the SNS ring vacuum test stand shown in Figure 1. It will be installed and used for SNS ring vacuum system commissioning at Oak Ridge National Laboratory (ORNL).

\section{SNS RING VACUUM SYSTEM}

\subsection{Vacuum System Overview}

The successful operation of SNS depends upon the reliable operation of the accelerator vacuum system in the high and ultra-high vacuum range under high-intensity beam operating conditions. Associated with the vacuum pressure levels are the availability and reliability requirements of the vacuum subsystems and their components. Three vacuum levels are required: less than $5 \times 10^{-8}$ Torr for the high-energy beam transport (HEBT) line to minimize the electron stripping of $\mathrm{H}^{-}$beam, less than $1 \times 10^{-8}$ Torr in the Ring to minimize the residual gas ionization, and below $1 \times 10^{-7}$ Torr for the ring to target beam transport (RTBT) line.

Due to the potential high radiation generated by the intense proton beam flux, the vacuum instrumentation will be situated at service buildings outside the accelerator tunnel whenever possible. There are three service buildings: one for HEBT, one for RTBT, and one for Ring subsystem equipment. The vacuum controllers are connected to their corresponding hardware through individual long cables, or to each other through multidrop serial links.

A major consideration in the design of ring vacuum

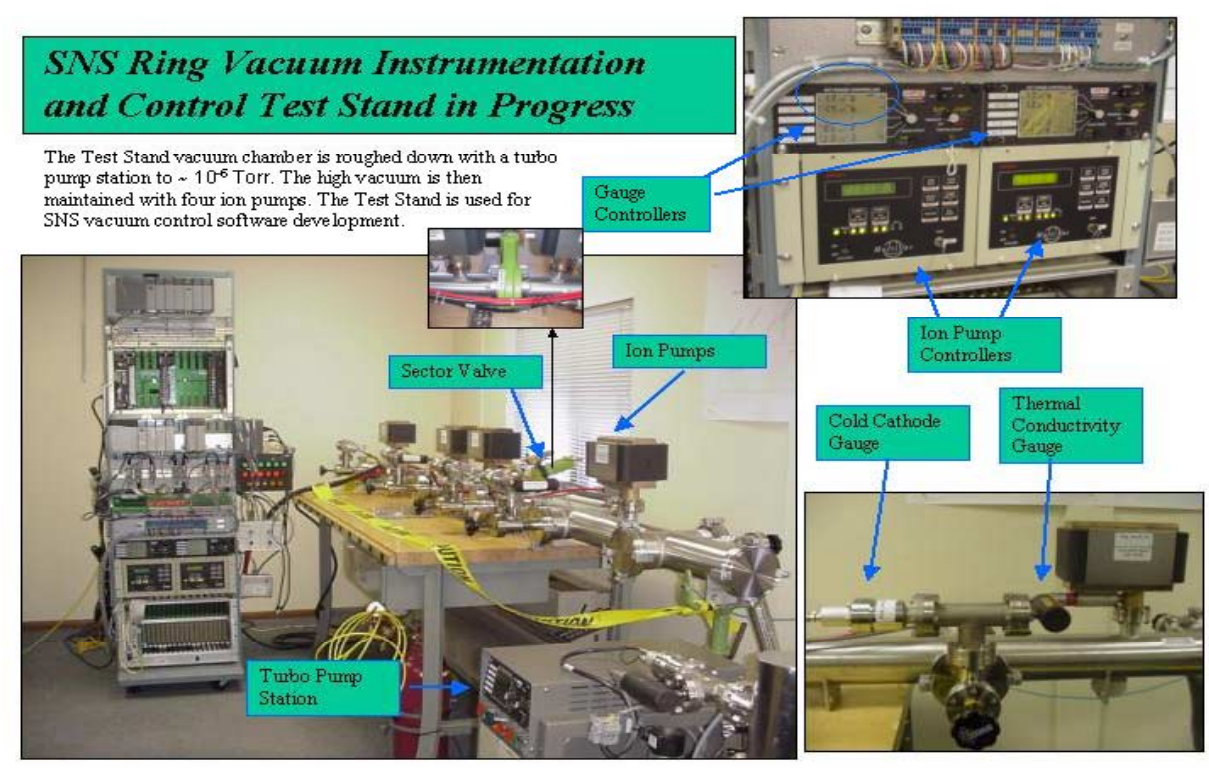

Figure 1. The SNS Vacuum Control Test Chamber.

\footnotetext{
$*$ Work performed under the auspices of the U.S. Department of Energy
+ jtang@bnl.gov
} 
instrumentation is standardization with other SNS vacuum systems, such as the ion source, the room temperature linac and the superconducting linac. The specification and operation modes of the vacuum instrumentation in these machine areas could be quite different than those of ring vacuum systems. Compromises have to be made in both specifications and cost to allow for the use of identical or similar device. The aim of standardization is to ease commissioning, maintenance and upgrade efforts, in addition to simplifying the control software development effort.

\subsection{Vacuum System Instrumentation}

Vacuum system instrumentation includes valves, gauges, ion pumps, turbomolecular pumps, and residual gas analyzers. All valves have $+24 \mathrm{Vdc}$ solenoids and have both open and closed limit switch-type indicators. Ion pumps will be used to maintain high vacuum in the accumulator ring and transport lines. Remote serial communication will be used to turn on/off the pump high voltage and to read pump current, pressure, and voltage. Convection-enhanced thermal conductivity gauges (TCGs) will be used to monitor the low vacuum levels within the accelerator, from 1000 to $10^{-4}$ Torr. The high vacuum levels within the accelerator, $10^{-3}$ to $10^{-10}$ Torr, will be measured using cold cathode gauges (CCGs). A multiple-gauge controller will control both CCGs and TCGs. Both ion pump and gauge controllers provide interlock inputs to the vacuum controls.

Partial pressure levels within the accelerator will be measured using residual gas analyzers (RGAs). The RGAs will be used to characterize the residual gases in the vacuum to aid in determining the gas source such as a water leak, an air leak or component outgassing.

Turbomolecular pump stations (TMPs) will be used to pump the beam line from atmospheric pressure to high vacuum, or to maintain vacuum in case of a leak. Remote operation of the TMP will be accomplished through remote control of analog inputs and discrete inputs and outputs.

\section{THE VACUUM I\&C SYSTEM}

\subsection{ControlLogix PLC for Vacuum Interlocks}

Allen-Bradley ControlLogix programmable logic controllers (PLCs) will be used to monitor gauge and pump setpoint outputs and control valves. The SNS Project has selected the ControlLogix PLC as the standard PLC [4]. Features of the ControlLogix system include being able to replace modules under power and read from all input modules from any processor within the ControlNet network.

The principal function of the PLC is to provide control of the sector gate valves that sectionalize the vacuum systems. The valve control logic will be fail-safe. A sector valve will close in case of a) vacuum conditions deteriorating to a specified limit, b) power loss, and c) operator input from the support building or remote terminal. The vacuum PLCs will also provide interlock outputs to SNS subsystems such as the RF System and other machine protection systems, and receive interlock inputs from subsystems such as the Target System. All vacuum system interlocks will use $24 \mathrm{Vdc}$ control power. Each PLC will monitor up to six 16-point input modules and control at least one 16-point output module.

A vote-to-close scheme will be implemented in the PLC ladder logic. A sector valve will close if any combination of two out of a possible three adjacent vacuum interlock signals indicate that the vacuum is above the acceptable operating limits. Each valve will have vote-to-close inputs from both upstream and downstream devices. The gauge and ion pump controllers will be used to conduct the

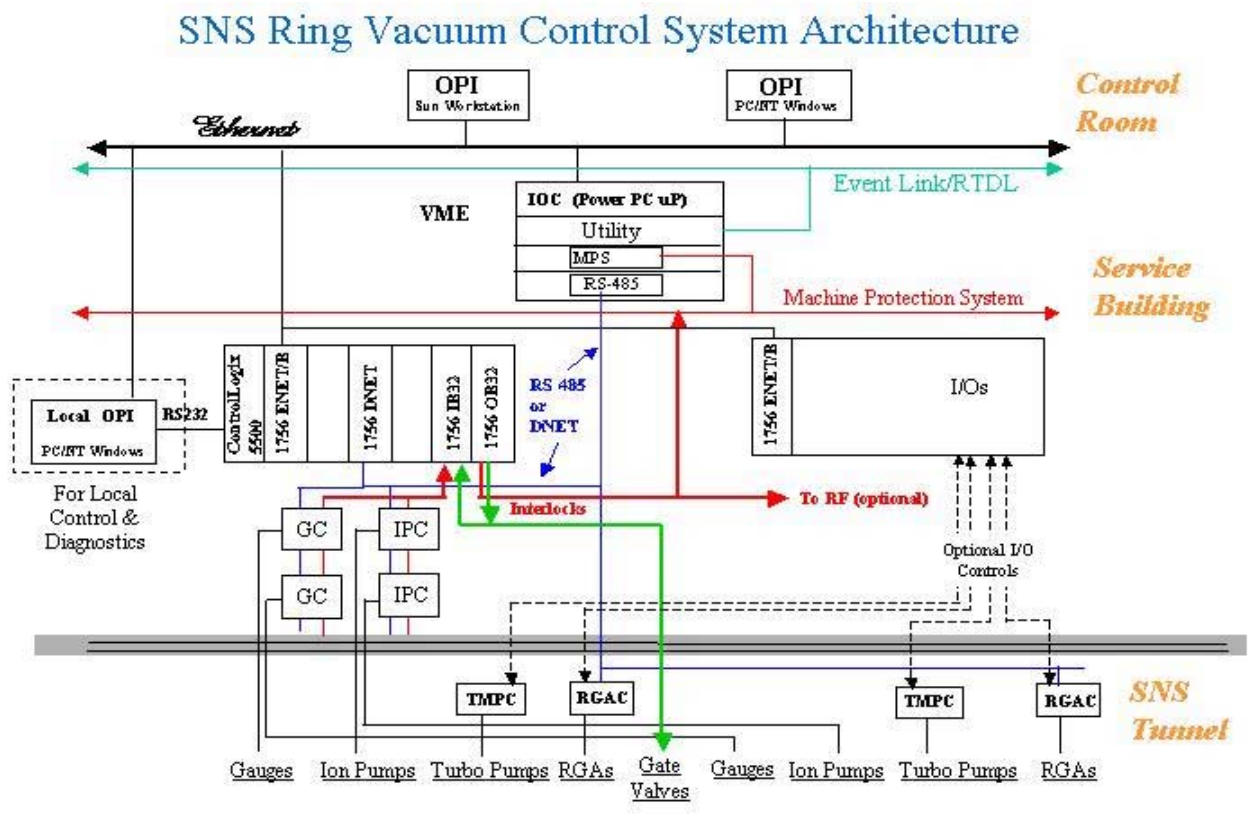

Figure 2. Proposed SNS Vacuum Control System Architecture. 
vacuum interlock signals. When the vacuum system is ready for beam operation, $+24 \mathrm{Vdc}$ is applied to the PLC input through relay contacts residing in the gauge and ion pump controllers. The relay contacts open if the pressure is above the threshold and if the controller or $24 \mathrm{Vdc}$ power supply lose input power. A given valve will not close if only one relay contact opens. The PLC ladder program, using set points from TCGs, prevents a sector gate valve from being opened if the vacuum on either side of the valve is at atmospheric pressure, since CCGs and ion pumps current readings might read zero current.

The control of the HEBT, Ring, and RTBT vacuum systems will be implemented using a network of PLCs. Four PLCs are planned, based on the vacuum device distribution: one each for the HEBT and RTBT subsystems, and two for the Ring subsystem. Data exchange between PLCs is accomplished through a realtime, redundant-media ControlNet network [5]. The highspeed, deterministic ControlNet network is ideal for transmitting time-critical vacuum system information and providing real-time control.

The PLCs in each service building will also generate machine protection system (MPS) beam permit signals appropriate for the various machine operation modes. Details of the MPS to vacuum system interface are yet to be determined.

\subsection{The Input/Output Controller}

The Input/Output Controller (IOC) is a VME controller where the control system core resides. The primary function of the IOC is to provide the gateway between the global control system and vacuum instrumentation system. All information for machine operators will be provided by the IOCs. Three IOCs are planned, one each for HEBT, Ring, and RTBT vacuum systems. The IOC will reside in a VME chassis located in the same support building as the PLC. The PLC may communicate with the IOC through an EtherNet/IP or ControlNet interface. The IOC may interface directly with vacuum device controllers if the vacuum device controllers use RS-485 serial communication.

\subsection{Operator Interface and Data Logging}

The Experimental Physics and Industrial Control System (EPICS) [6] will be used to provide the graphical user interface for operation of the vacuum systems via the IOC. An EPICS display manager will be used for monitoring and controlling vacuum instrumentation. A typical graphic operator interface (OPI) screen for SNS vacuum controls is shown in Figure 3 . The display manager will be able to run on Unix and Windows NT platforms.

The vacuum system display will be updated in five-tothirty second intervals, depending upon device response

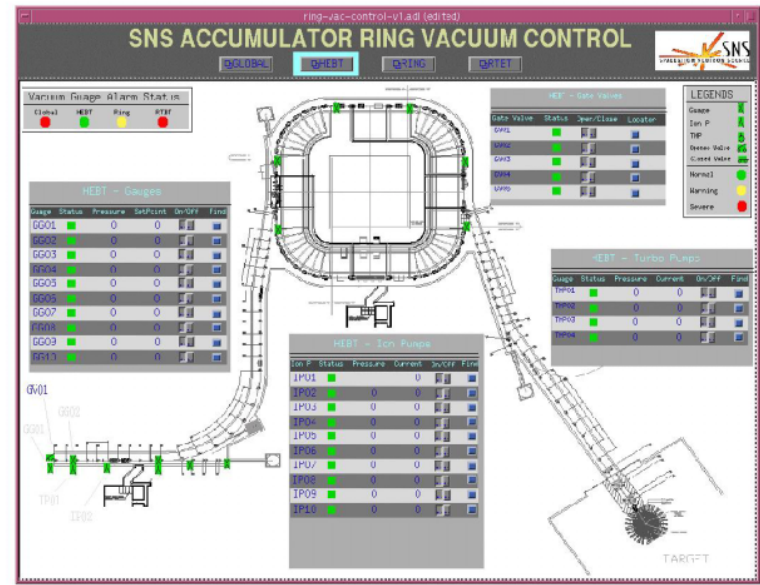

Figure 3. A typical OPI screen for SNS vacuum control.

time and subsystem requirements. Beam vacuum gauge readings will likely be updated more frequently than ion pump readings. The control system will provide data logging applications and tools to create, edit, save, and view data logging files. Readings from collections of vacuum devices will be logged at intervals ranging from ten seconds to one hour.

\section{SUMMARY}

The SNS Ring vacuum instrumentation and control are based on RHIC vacuum instrumentation architecture and EPICS control system, both are proven at existing accelerator vacuum systems. The specifications for vacuum instrumentation are been drafted and will be purchased in the coming year. The PLC ladder logic programming for SNS ring vacuum valve interlocks will also be completed this year. The SNS Ring vacuum control system [7] will be ready for commissioning in 2004 at ORNL.

\section{REFERENCES}

[1] W. T. Weng, "Accumulator Ring Design for SNS Project”, PAC'97, Vancouver, p970 (1997).

[2] H. C. Hseuh, C.J. Liaw and M. Mapes, "Design of the SNS Accumulator Ring Vacuum Systems", PAC'99, New York, p1345, (1999).

[3] R.C. Lee, et al., "RHIC Vacuum Instrumentation and Control System", PAC'99, New York, p1348, (1999).

[4] J.Y. Tang, et al. "A Distributed and Collaborative PLC lab for SNS”, ICALEPCS'99, Trieste, Italy, p370 (1999)

[5] http://www.controlnet.org/

[6] L. R. Dalesio, et al., "EPICS", Nuclear Instruments \& Methods in Physics Research A 352 (1994), 179-184.

[7] http://www.sns.bnl.gov/vacuum/ 